\title{
THE GRAPHS OF SEMIRINGS. II
}

\author{
J. S. RATTI AND Y.-F. LIN
}

ABSTRACT. In this paper the authors continue their investigation of the connectivity of the graphs of semirings. We give some sufficient conditions under which the graph of a semiring is connected. The paper also contains some open problems.

1. Introduction. In [1], [2], and [4] the authors studied the graphs of semirings, and gave a few sufficient conditions for a semiring to have connected graph. To make this paper self contained, we first review some of the basic definitions.

A semiring is a nonempty set $R$ equipped with two binary operations, called addition, + , and multiplication (denoted by juxtaposition), such that $R$ is multiplicatively a semigroup and additively a commutative semigroup, and that the multiplication is distributive across the addition both from the left and from the right. We note that the existence of a zero element in a semiring is not assumed.

By the graph $G(R)$ of a semiring $R$, we mean the nonoriented graph whose vertices consist of the set $R$ of all proper subsemirings of $R$, and two vertices $V_{1}$ and $V_{2}$ are adjacent (or joined by an edge) if and only if $V_{1} \cap V_{2} \neq \varnothing$. The graph $G(R)$ is said to be connected if and only if for each pair of vertices $V$ and $V^{\prime}$ there exists a finite sequence, called a path, of vertices $V=V_{1}, V_{2}, \cdots, V_{n}=V^{\prime}$ such that every two consecutive vertices are adjacent.

The distance $d\left(V_{\alpha}, V_{\beta}\right)$ between two vertices $V_{\alpha}$ and $V_{\beta}$ of a graph is the number of edges in a shortest path between these vertices (if no such path exists, we define $d\left(V_{\alpha}, V_{\beta}\right)=+\infty$; of course $\left.d\left(V_{\alpha}, V_{\alpha}\right)=0\right)$. The diameter of a graph is the supremum of $d\left(V_{\alpha}, V_{\beta}\right)$, where $\left(V_{\alpha}, V_{\beta}\right)$ runs over all pairs of vertices of the graph.

In [1], the authors posed the following conjecture: "The graph $G(R)$ of a semiring $R$ of cardinality distinct from two is connected." Although this conjecture still remains unsolved, we are now able to prove it for the following four classes of semirings:

(1) Semirings with a multiplicative idempotent element.

(2) Additively cancellative semirings.

Presented to the Society, January 22, 1971; received by the editors December 16, 1970.

AMS 1970 subject classifications. Primary 05C25, 16A78.

Key words and phrases. Semiring, graph, connectivity of a graph, diameter of a graph, left unital semiring, cancellative semiring, disconnected graphs. 
(3) Multiplicatively cancellative semirings.

(4) Semirings with an additive zero.

In the last section of the paper, we pose some open questions. Solution of any of these questions will be of interest and perhaps contribute to the complete solution of the conjecture mentioned above.

2. Connectivity theorems. Throughout this section we assume that a semiring has more than two elements. The semirings having exactly two elements may have a disconnected graph and hence will be discussed separately in the next section. In [1] the authors proved the following:

THEOREM A. The graph of a left unital semiring having more than two elements is connected.

This theorem will be used in the proofs of the connectivity theorems of this section.

THEOREM 1. The graph of a semiring with an idempotent element is connected.

Proof. Let $R$ be such a semiring and let $e$ be an idempotent element, that is $e^{2}=e$, of $R$. Let $A$ and $B$ be two disjoint proper subsemirings of $R$, and let $a \in A$ and $b \in B$ be two fixed elements. We first observe that $\{A, a R, R b, B\}$ is a path connecting $A$ and $B$, unless $a R=R$ or $R b=R$. Assume $a R=R$ (the case $R b=R$ may be similarly handled). We now divide the rest of proof into the following cases:

Case 1. $R a=R$.

This together with $a R=R$ implies that $R$ is left unital and hence by Theorem A, the graph of $R$ is connected.

Case 2. $R a \neq R$.

Suppose that $b R \neq R$, then $\{A, R a, b R, B\}$ would be a path connecting $A$ and $B$. Now consider the case $b R=R$. If $R b=R$ then it follows, as Case 1 above, that the graph of $R$ is connected. Assume $R b \neq R$. Then $\{A, R a, x R, R b, B\}$ would be a path connecting $A$ and $B$, unless $x R=R$ for all $x \in R$. In the latter case, $e R=R$ and hence $R$ is left unital. By Theorem A again, the graph of $R$ is connected.

This exhausts all the possibilities, and the proof for Theorem 1 is now complete.

A semiring $R$ is said to be additively cancellative if and only if $x+c=y+c$ in $R$ implies $x=y$.

THEOREM 2. The graph of an additively cancellative semiring is connected. 
Proof. Let $A$ and $B$ be any two disjoint proper subsemirings of an additively cancellative semiring $R$, and let $a \in A$ and $b \in B$ be any two fixed elements. Then as in the proof of Theorem 1 above, we may assume without loss of generality that $a R=R, b R=R, R a \neq R \neq R b$. Since $R a \cup(R a+R b) \cup R b$ is a subsemiring of $R$ intersecting both $A$ and $B,\{A, R a \cup(R a+R b) \cup R b, B\}$ would be a path connecting $A$ and $B$, unless $R a \cup(R a+R b) \cup R b=R$. Now we assume further that $R a \cup(R a+R b) \cup R b=R$. It follows that we may assume $a$, $b \in R a+R b$; for, if otherwise we would have $a$ or $b \in R a \cup R b$. If $a \in R a$ then there exists $d \in R$ such that $a=d a$, which together with $a R=R$ implies that $R$ is left unital. Hence by Theorem $\mathrm{A}$, the graph of $R$ is connected. If $a \in R b$ then $\{A, R b, B\}$ forms a path connecting $A$ and $B$. The other case $b \in R a \cup R b$ leads to the same conclusion.

The case $a, b \in R a+R b$ implies that $\{A, R a+R b, B\}$ would be a path connecting $A$ and $B$, unless $R a+R b=R$. Let $R a+R b=R$, and let $C=\{z \mid x a+z \in R a$ for some $x \in R\}$. Then it can be verified that $C$ is a subsemiring containing $R a$. Since $a^{2} \in C$, we have $A \cap C \neq \varnothing$. Also, for any $r \in R, r a \in R=R a+R b$ implies there exist $s, t \in R$ such that $s a+t b=r a$ which shows $t b \in R b \cap C \neq \varnothing$. Consequently, $\{A, C, R b, B\}$ would be a path connecting $A$ and $B$, unless $C=R$. Suppose $C=R$. Since $a R=R$, there exists an element $e \in R$ such that $a e=a$. It follows from $e \in C$ that there exist $u$ and $v \in R$ such that $u a+e=v a$. Multiplying $e$ from the right to each term of the last equality, we have $u a+e^{2}=v a$. We now have $u a+e^{2}=u a+e$ which implies $e^{2}=e$, by the additive cancellation. Thus, $R$ has an idempotent and hence, by Theorem 1 , the graph of $R$ is connected.

A semiring $R$ is said to be cancellative provided that $x c=y c$ in $R$ implies $x=y$, and that $c x=c y$ implies $x=y$.

THEOREM 3. The graph of a cancellative semiring is connected.

Proof. Let $R$ be a cancellative semiring. Let $A$ and $B$ be two disjoint proper subsemirings of $R$, and let $a \in A$ and $b \in B$ be two fixed elements. As in the proof of Theorem 1 above, one may assume without loss of generality that either $a R=R$ or $R b=R$.

Case 1. $a R=R$.

It follows that there exists an element $e \in R$ such that $a e=a$, and hence $a e^{2}=a e$. The semiring $R$ is cancellative implies that $e^{2}=e$. Hence $e$ is an idempotent element. By Theorem 1, the graph of $R$ is connected.

Case 2. $R b=R$.

The proof for this case is similar to that of Case 1 , above.

An additive zero of a semiring $R$ is an element $z \in R$ such that $x+z=x$ 
for all $x \in R$. An additive zero element need not be multiplicatively zero, and a semiring need not have an additive zero. It is obvious that if a semiring has an additive zero element, it is unique.

THEOREM 4. The graph of a semiring with an additive zero is connected.

Proof. Let $R$ be a semiring with an additive zero $z$. As in the proof of Theorem 1, we may assume without loss of generality that $x R=R$ for all $x \in R$. In particular, we have $z R=R$. We show that $z^{2}=z$ and hence by Theorem 1 the graph of $R$ is connected. To this end, let $x$ be any arbitrary element of $R$. Then $z R=R$ implies that there exists an element $x^{\prime} \in R$ such that $x=z x^{\prime}$. Whence,

$$
x+z^{2}=z x^{\prime}+2^{2}=z\left(x^{\prime}+z\right)=z x^{\prime}=x,
$$

and hence, by the uniqueness of additive zero element, we have $z^{2}=z$. The proof is now complete.

3. Examples of semirings with disconnected graphs. Since there are only four distinct semigroups with two elements (excluding isomorphic and anti-isomorphic ones) of which three are commutative [3], there can at most be $4 \times 3=12$ distinct semirings with two elements. We checked each one of these and discovered that there are exactly five distinct semirings with two elements that have disconnected graph; we list the addition and multiplication tables of these five exceptional semirings below:

NUMBER 1.

\begin{tabular}{l|ll}
$\cdot$ & 0 & 1 \\
\hline 0 & 0 & 0 \\
1 & 0 & 1
\end{tabular}

\begin{tabular}{l|ll}
+ & 0 & 1 \\
\hline 0 & 0 & 1 \\
1 & 1 & 1
\end{tabular}

Number 2.

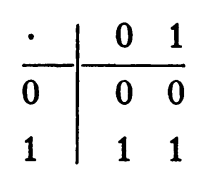

\begin{tabular}{l|ll}
+ & 0 & 1 \\
\hline 0 & 0 & 0 \\
1 & 0 & 1
\end{tabular}

Number 3.

\begin{tabular}{l|ll}
$\cdot$ & 0 & 1 \\
\hline 0 & 0 & 0 \\
1 & 0 & 1
\end{tabular}

\begin{tabular}{l|ll}
+ & 0 & 1 \\
\hline 0 & 0 & 0 \\
1 & 0 & 1
\end{tabular}


NUMBER 4.

\begin{tabular}{l|ll}
$\cdot$ & 0 & 1 \\
\hline 0 & 0 & 0 \\
1 & 1 & 1
\end{tabular}

\begin{tabular}{l|ll}
+ & 0 & 1 \\
\hline 0 & 0 & 1 \\
1 & 1 & 1
\end{tabular}

NUMBER 5.

\begin{tabular}{l|ll}
$\cdot$ & 0 & 1 \\
\hline 0 & 0 & 1 \\
1 & 1 & 1
\end{tabular}

\begin{tabular}{l|ll}
+ & 0 & 1 \\
\hline 0 & 0 & 0 \\
1 & 0 & 1
\end{tabular}

In $[1$, p. 76$]$, it was erroneously conjectured that the example Number 1 would be the only semiring with disconnected graph; now we have five such semirings all of which have two elements.

4. Open problems. It is conjectured in [4] that the graph of an arbitrary semiring with more than two elements is connected and the diameter of the graph does not exceed three. This problem seems rather difficult; we now ask the same question for some special situations:

In what follows, let $R$ always be an arbitrary semiring with more than two elements.

PROBLEM 1. Suppose that, for some $x_{0} \in R$, the graph of $R x_{0}$ is connected. Is the graph of $R$ connected? What is the diameter of the graph of $R$ ?

Even the answer to the following more restricted case of Problem 1 seems to be unknown:

Problem 2. Let $R$ be such that every proper subsemiring has a connected graph. Is the graph of $R$ connected? If the answer is affirmative, is the diameter of the graph less than or equal to three?

Problem 3. Let $R$ be a semiring such that the diameter of the graph of every proper subsemiring does not exceed $k$. What can be said about the diameter of $R$ ?

Problem 4. Suppose that $R$ satisfies either the right (or left) one side multiplicative cancellation law. Is the graph of such an $R$ connected?

Problem 5. Let $R$ be a semiring such that $x+x=x$ for one or for every $x \in R$. Is the graph of such an $R$ connected?

Problem 6. In the proof of Theorem 1, above, it is easy to see that the diameter of the graph of $R$ (with an idempotent) does not exceed four. Is it true that the diameter of the graph of $R$ in this theorem does not exceed three? 
Problem 7. Given a connected graph $G$ with diameter less than or equal to three, does there exist a semiring $R$ such that $G$ is the graph of $R$ ?

\section{REFERENCES}

1. Y.-F. Lin and J. S. Ratti, The graphs of semirings, J. Algebra 14 (1970), 73-82.

2. - Connectivity of the graphs of semirings: lifting and product, Proc. Amer. Math. Soc. 24 (1970), 411-414.

3. George E. Forsythe, SWAC computes 126 distinct semigroups of order 4, Proc. Amer. Math. Soc. 6 (1955), 443-447. MR 16, 1085.

4. J. S. Ratti and Y.-F. Lin, The diameters of the graphs of semirings, J. Austral. Math. Soc. 11(1970), 433-440.

University of South Florida, Tampa, Florida 33620 